\title{
On a problem of Andersson and Perlman
}

\author{
M.A. Steel and G.R. Wood \\ Department of Mathematics, University of Canterbury, Christchurch, New Zealand
}

Received November 1992

Abstract: We present a simple solution to a problem posed recently by Andersson and Perlman. This solution allows us to find the conditional independence assumptions necessary to permit maximum likelihood estimation of the parameters of a multivariate normal distribution, when certain observations are missing.

Keywords: Multivariate normal; parameter estimation; missing data; lattice; join-irreducible.

\section{Introduction}

Andersson and Perlman (1991) considered the problem of maximum likelihood estimation of the parameters of a $p$-variate normal distribution, based on an independent sample $x_{1}, \ldots, x_{n}$, some of which have missing components. If the missing components are not nested, then the maximum likelihood estimators cannot be obtained in explicit form. In order to obtain such estimators explicitly they show that it is necessary to impose a minimal set of conditional independence assumptions. These assumptions are determined by the join-irreducible elements in a finite distributive lattice, the lattice itself being determined by the missing data pattern. Andersson and Perlman (1991, p. 486) state that it would be desirable to find an algorithm, polynomial in $p$, which would find these join-irreducible elements. The purpose of this note is to describe these elements, and hence reveal that a best polynomial time algorithm is available, polynomial however in $p$ and $m$. Here $m$ is the cardinality of $\mathscr{S}$, where $\mathscr{S}$ is defined below. Since $m$ can be as large as $2^{p}$, there can exist no algorithm polynomial only in $p$.

Correspondence to: Dr. G.R. Wood, Department of Mathematics, University of Canterbury, Christchurch, New Zealand.

\section{Results}

Consider the missing data pattern shown below given in Andersson and Perlman (1991, Example 4.13) for $p=5$ variables, and a sample of size $n=3$. The ' $i$ ' indicates that component $i$ of that observation is present, while a blank indicates a missing observation.

$$
\begin{array}{lll}
1 & 1 & 1 \\
& 2 & 2 \\
3 & & \\
& 4 & \\
& & 5
\end{array}
$$

If we let $I=\{1,2,3,4,5\}$, Andersson and Perlman summarise this pattern using the subset $\mathscr{S}$ of $2^{I}$ determined by the columns, namely

$\mathscr{S}=\{\{1,3\},\{1,2,4\},\{1,2,5\}\}$,

so here $m=3$. Now $\mathscr{S}$ determines a distributive sublattice $\mathscr{K}$ of $2^{I}$, being all finite unions of finite intersections of elements of $\mathscr{S}$. In turn, $\mathscr{K}$ contains join-irreducible elements, $J(\mathscr{K})$. These are elements $A$ in $\mathscr{K}$ such that if $A=B \cup C$, with $B$ and $C$ in $\mathscr{K}$, then $A=B$ or $A=C$. In this example we find that

$J(\mathscr{K})=\{\{1\},\{1,2\},\{1,3\},\{1,2,4\},\{1,2,5\}\}$. 
From this, Andersson and Perlman deduce that the required conditional independence assumptions are

$x_{2} \Perp x_{3} \mid x_{1} \quad$ and $\quad x_{3} \Perp x_{4} \Perp x_{5} \mid\left(x_{1}, x_{2}\right)$.

In the sequel, $I=\{1,2, \ldots, p\}, \mathscr{P}$ is a subset of $2^{I}$ of cardinality $m$ with $\cup \mathscr{S}=I, \mathscr{K}$ is the distributive lattice generated by $\mathscr{S}$ and $J(\mathscr{K})$ is the set of join-irreducible elements in $\mathscr{K}$. It is easily verified that $\mathscr{K}=\bigcup[\cap \mathscr{S}]$. For each $i \in I$, let $\mathscr{F}_{i}=\{A \in \mathscr{S}: i \in A\}$.

The lemma following characterises elements of $\mathscr{K}$, and the theorem characterises elements of $J(\mathscr{K})$. The corollary answers the question of Andersson and Perlman.

Lemma 1. $B \in \mathscr{K}$ if and only if $B=\cup_{i \in B}\left[\cap \mathscr{F}_{i}\right]$.

Proof. The sufficiency is immediate, since $\mathscr{K}=$ $\cup[\cap \mathscr{S}]$. Suppose $B \in \mathscr{K}$. To show that $B \subseteq$ $\bigcup_{i \in B}\left[\cap \mathscr{F}_{i}\right]$, consider $j \in B$. Then since $j \in$ $\cap \mathscr{F}_{j}$, certainly $j \in \bigcup_{i \in B}\left[\cap \mathscr{F}_{i}\right]$. To show that $\bigcup_{i \in B}\left[\cap \mathscr{F}_{i}\right] \subseteq B$, note that if $i \in B$, then $\cap \mathscr{F}_{i} \subseteq$ $B$, since $B \in \mathscr{K}$.

Theorem 1. $J(\mathscr{K})=\left\{\cap \mathscr{F}_{i}: i \in I\right\}$.

Proof. Take $B \in J(\mathscr{K})$. From the lemma we know that $B$ has the form $\cup_{i \in B}\left[\cap \mathscr{F}_{i}\right]$. If $B$ is also join-irreducible it must be of the form $\cap \mathscr{F}_{i}$, for some $i \in I$. We now show that each set of the form $\cap \mathscr{F}_{i}$ is join-irreducible. Suppose $\cap \mathscr{F}_{i}=$ $B \cup C$, for $B, C \in \mathscr{K}$. Then $i$ must lie in one of $B$ or $C, B$ say. Thus $\cap \mathscr{F}_{i} \subseteq B$ so $B=\bigcap \mathscr{F}_{i}$, and so $\cap \mathscr{F}_{i}$ is join-irreducible.

Corollary 1. An algorithm to construct $J(\mathscr{K})$ directly from $\mathscr{S}$ is available, with time complexity polynomial in $m$ and $p$.

Proof. From the theorem we know that $J(\mathscr{K})=$ $\left\{\cap \mathscr{F}_{i}: i \in I\right\}$. Each $\cap \mathscr{F}_{i}$ can be constructed in $\mathrm{O}(m p)$ operations, so we can find $J(\mathscr{K})$ in $\mathrm{O}\left(m p^{2}\right)$ operations.

We conclude by returning to the earlier example. Note that $\cap \mathscr{F}_{1}=\{1\}, \cap \mathscr{F}_{2}=\{1,2\}, \cap \mathscr{F}_{3}=$ $\{1,3\}, \cap \mathscr{F}_{4}=\{1,2,4\}$ and $\cap \mathscr{F}_{5}=\{1,2,5\}$, precisely the join-irreducible sets $J(\mathscr{K})$.

\section{Reference}

Andersson, S.A. and M.D. Perlman (1991), Lattice-ordered conditional independence models for missing data, Statist. Probab. Lett. 12, 465-486. 